Article

\title{
Improving Performance of a Resonant String-Based Pulsation Attenuator in Hydraulic Systems
}

\author{
Xia Shang, Hua Zhou * and Huayong Yang \\ State Key Laboratory of Fluid Power and Mechatronic Systems, Zhejiang University, Hangzhou 310027, China; \\ shangxia1007@163.com (X.S.); yhy@zju.edu.cn (H.Y.) \\ * Correspondence: hzhou@zju.edu.cn
}

Received: 17 October 2020; Accepted: 27 November 2020; Published: 28 November 2020

\begin{abstract}
Hydraulic pulsation attenuators (HPA) are commonly used to suppress the noise and vibration in fluid power systems. However, most existing HPAs lack a reasonable optimization strategy and effective methods to improve the performance of HPAs. This paper proposes an adaptive particle swarm optimization (APSO) algorithm to speed up the geometry optimization process of a resonant string-based compound HPA (RSHPA), which was proposed in our previous work. Then, the study discusses the possibility of improving the performance of RSHPA by varying the configuration and location of the RSHPA. The experiment result validates the feasibility of the proposed optimization method for RSHPA.
\end{abstract}

Keywords: resonant string; pulsation attenuator; hydraulic system; flow ripple; optimization

\section{Introduction}

Compact, easy-to-tune and affordable hydraulic pulsation attenuators (HPAs) are commonly used for damping the noise and vibration in fluid power systems. The passive-type HPAs are preferred devices for most engineers to solve the pulsation problems because they do not need controllers to tune the operational modes and have a simple structure for fabrication. The typical passive HPAs include the Helmholtz resonator [1], expansion chamber [2], perforated-pipe silencer [3], and fluid-structure interaction silencers [4].

The geometry optimization method is commonly used in improving the performance of mechatronic devices [5-7], including the HPAs. Researchers used different optimization algorithms, such as the genetic algorithm (GA) [8], particle swarm optimization (PSO) and the traversal search method (TSM) [9]. GA and PSO prove to be effective in most optimization cases. However, as the number of geometry parameters needed to be optimized increases, the consuming time of the optimization process would increase rapidly. TSM can guarantee that the optimized results are globally optimal, but its consuming time is much more than traditional optimization algorithms (GA and PSO). Therefore, this paper proposes an adaptive particle swarm optimization (APSO) method to improve the computational efficiency of the optimization process.

In this paper, the optimization object is the resonant string-based HPA (RSHPA), which was proposed in our previous work [10]. The trend of using HPAs is moving from constant speed propulsion of the pumps to the variable speed, which requires the attenuators to be designed for a wider frequency range. Nevertheless, the attenuators that can suppress the noise at a specified narrow frequency band are still very useful for some critical operating conditions (e.g., constant load conditions). In addition, they generally have better attenuation strength at a specified operating point than that with wide-band attenuation performance under the same bulk.

Conventional optimization strategy concentrates on optimizing geometry parameters of devices. However, the geometry parameter is not the only factor affecting the performance of the HPA. 
The configuration and installation position also decide the performance of the HPA, but they are rarely discussed in the existing studies. Thus, in addition to optimizing the geometry, investigating the possible configuration and finding an appropriate installation position of RSHPA in a hydraulic system are also presented in this paper.

The rest of this paper is organized as follows. Section 2 simply reviews the numerical model of our previous work and introduces the evaluation method of the RSHPA. Section 3 introduces the APSO algorithm and its numerical tests. Section 4 introduces the optimization method of RSHPA. Finally, Section 5 draws a conclusion and discusses future work.

\section{Modelling of the Resonant String-Based Hydraulic Pulsation Attenuator (RSHPA)}

\subsection{Modelling}

Figure 1 demonstrates a 3-dimensional model of the RSHPA [10]. The structure is composed of an expansion chamber and multiple parallel strings. The strings can be resonant with the fluid pulsation whose frequency is equal to its natural resonant frequency. Then the strings absorb the pulsation energy and transform it to the vibration, which finally becomes the friction heat energy. The expansion chamber suppresses the pulsation by neutralizing the transmitting and reflecting waves via the abrupt cross-sectional area. Thus, RSHPA has potential to work for both wide band and specified frequency conditions. In this paper, the RSHPA is installed in a typical hydraulic system, as shown in Figure 2 where the power source and load are a pump and a throttle valve, respectively. The uppercase and lowercase letters of the pressure and flow represent the parameters used at the frequency and time domains, respectively.

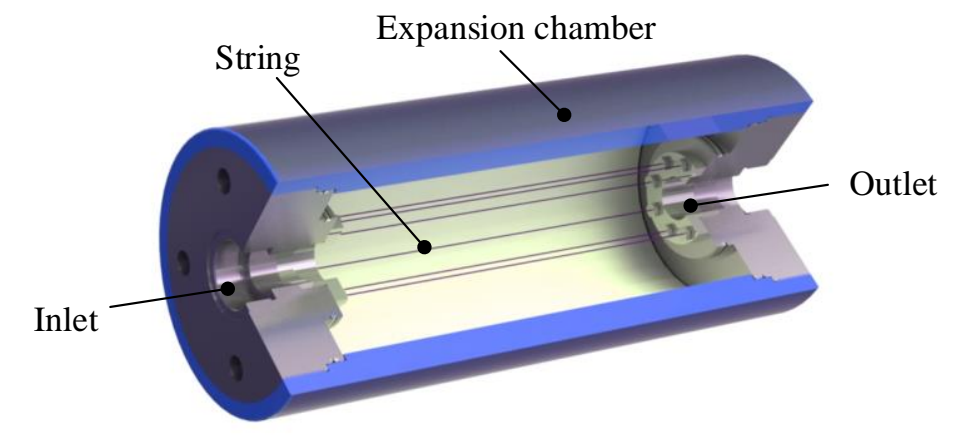

Figure 1. 3D model of the RSHPA.

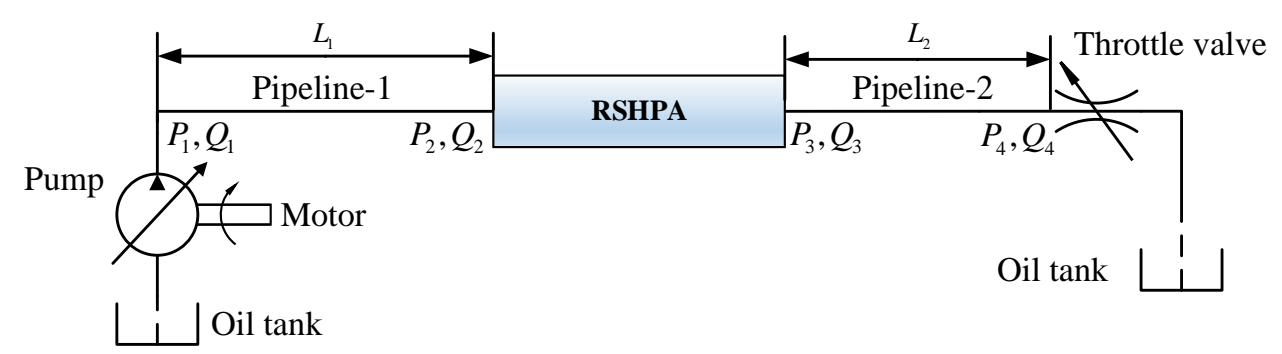

Figure 2. Schematic diagram of a hydraulic system with the resonant string-based hydraulic pulsation attenuator (RSHPA).

The transfer matrix of the upstream pipeline, RSHPA and the downstream pipeline are defined as $\mathbf{A}_{1}, \mathbf{A}_{2}$ and $\mathbf{A}_{3}$, respectively. The transfer matrix of pipelines can be obtained by taking Laplace transform of their dynamic functions. The detailed derivation can refer to the literature [10]. Here, we provide the transfer matrix of pipelines directly as follows. 


$$
\mathbf{A}_{1}=\mathbf{A}_{3}=\left[\begin{array}{cc}
\operatorname{ch} \Gamma(s) & Z_{\mathrm{c}}(s) \operatorname{sh} \Gamma(s) \\
\frac{\operatorname{sh} \Gamma(s)}{Z_{\mathrm{c}}(s)} & \operatorname{ch} \Gamma(s)
\end{array}\right]
$$

where $\operatorname{sh}(\cdot)$ and $\operatorname{ch}(\cdot)$ denote the hyperbolic sine and cosine functions, $s$ denotes the complex variable. To analyze the characteristics of RSHPA at the frequency domain, the complex number $s$ can be substituted by $s=j \omega$ where $\omega$ denotes the angular frequency of the system. The angular frequency can be calculated by $\omega=2 \pi f$ where $f$ denotes the system frequency. $\Gamma(s)$ denotes the propagation operator, and $Z_{\mathrm{c}}(s)$ denotes the characteristic impedance of the pipeline. $\Gamma(s)$ and $Z_{\mathrm{c}}(s)$ are given as [11]:

$$
\begin{gathered}
\Gamma(s)=\frac{s L_{i}}{c_{0}} \sqrt{1+\frac{8 \pi v_{0}}{A_{p} s}} \\
Z_{\mathrm{c}}(s)=\frac{\rho_{0} c_{0}}{A_{p}} \sqrt{1+\frac{8 \pi v_{0}}{A_{p} s}} .
\end{gathered}
$$

where $L_{i}(i=1,2)$ denotes the length of the pipeline, $v_{0}$ denotes the kinematic viscosity of the fluid, $A_{p}=\pi d^{2} / 4$ denotes the sectional area of the pipeline, $d$ denotes the inner diameter of the pipeline, $\rho_{0}$ denotes the density of the fluid, and $c_{0}$ denotes the speed of wave propagation in fluid. Note that the propagation of the fluid satisfies the plane wave condition and the viscous friction loss of the fluid is consequently assumed to be proportional to the fluid speed [11].

For the RSHPA, the transfer matrix is calculated as [10]

$$
\mathbf{A}_{2}=\left[\begin{array}{cc}
1+\frac{\left(Z_{\left.C_{E C A}+Z_{\text {string }}\right)\left(R_{E C A}+Z_{L_{E C A}}\right)} Z_{C_{E C A}} Z_{\text {string }}\right.}{Z_{C_{E C A}+Z_{\text {string }}}} & R_{E C A}+Z_{L_{E C A}} \\
\frac{Z_{C_{E C A}} Z_{\text {string }}}{2} & 1
\end{array}\right]
$$

where $R_{E C A}, Z_{C_{E C A}}$ and $Z_{L_{E C A}}$ denote the equivalent resistance, capacitive impedance and inductive impedance of the expansion chamber, respectively, and $Z_{\text {string }}$ denotes the equivalent impedance of the string array. It is noted that the derivation method of the attenuator model is essentially a lumped-parameter method, which believes that each hydraulic element, such as a pipeline, can be electrically modelled by a combination of a resistance, inductance and a capacitance. This method is accurate enough under the assumption that the fluid in the pipeline is laminar flow and the major pulsation frequency is within a certain range, which would not heavily affect the impedance of the hydraulic elements [12].

These parameters are calculated as [10]:

$$
\begin{gathered}
R_{E C A}=\frac{128 \mu_{0} l}{\pi D^{4}}, Z_{C_{E C A}}=-j \frac{4 \rho_{0} c_{0}^{2}}{\omega \pi l D^{2}}, Z_{L_{E C A}}=\frac{4 j \omega \rho_{0} l}{\pi D^{2}} \\
Z_{\text {string }}=Z_{1} / / Z_{2} / / \ldots / / Z_{i} / / \ldots / / Z_{g}
\end{gathered}
$$

where $\mu_{0}$ denotes the kinetic viscosity of the fluid, $l$ and $D$ denote the length and diameter of the expansion chamber, $Z_{i}$ denotes the impedance of the $i$-th string and "//" denotes the parallel operator for the impedance. The equivalent circuit diagram of the string array is illustrated as Figure 3. Each string is composed of infinite resonant LC (Inductance-Capacitance) units, which represent the response characteristics of different-order frequency of the string. 


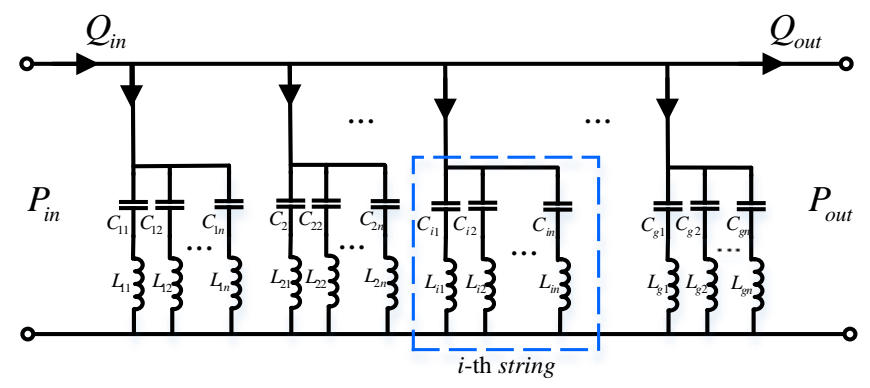

Figure 3. Equivalent circuit of the string array in the RSHPA.

The resonant frequency for each component is formulized as follows [13,14]:

$$
f_{\text {in }}=1 / 2 \pi \sqrt{L_{i n} C_{i n}}
$$

where $L_{i n}$ and $C_{i n}$ denote the equivalent inductance and capacitance of the $n$-th order response of the $i$-th string. Based on Figure 3, the equivalent impedance of $i$-th string is given by:

$$
Z_{i}=\left(\frac{1}{j \omega C_{i 1}}+j \omega L_{i 1}\right) / /\left(\frac{1}{j \omega C_{i 2}}+j \omega L_{i 2}\right) / / \cdots / /\left(\frac{1}{j \omega C_{i n}}+j \omega L_{i n}\right), n=1,2, \cdots .
$$

where [15],

$$
L_{\text {in }}=\frac{\rho_{0} l_{s t r}^{i}}{\pi\left(\Theta_{i} / 2\right)^{2}} .
$$

In Equation (9), $l_{\text {str }}^{i}$ and $\Theta_{i}$ denote the length and diameter of the $i$-th string, respectively. The equivalent capacitance in Equation (8) can be calculated using Equation (7). With Equations (1)-(9), the transfer matrix of the RSHPA can be determined.

\subsection{Performance Evaluation Method}

The hydraulic system shown in Figure 2 can be expressed by:

$$
\left[\begin{array}{c}
P_{1}(s) \\
Q_{1}(s)
\end{array}\right]=\mathbf{A}_{1} \mathbf{A}_{2} \mathbf{A}_{3}\left[\begin{array}{c}
P_{4}(s) \\
Q_{4}(s)
\end{array}\right]=\left[\begin{array}{ll}
a_{11} & a_{12} \\
a_{21} & a_{22}
\end{array}\right]\left[\begin{array}{c}
P_{4}(s) \\
Q_{4}(s)
\end{array}\right]
$$

where $a_{11} \sim a_{22}$ denote four elements of the total transfer matrix of the system. If the RSHPA is replaced by a pipeline, the system can be expressed by:

$$
\left[\begin{array}{l}
P_{1}^{\prime}(s) \\
Q_{1}^{\prime}(s)
\end{array}\right]=\mathbf{A}_{1} \mathbf{A}_{22}^{\prime} \mathbf{A}_{3}\left[\begin{array}{c}
P_{4}^{\prime}(s) \\
Q_{4}^{\prime}(s)
\end{array}\right]=\left[\begin{array}{ll}
a_{11}^{\prime} & a_{12}^{\prime} \\
a_{21}^{\prime} & a_{22}^{\prime}
\end{array}\right]\left[\begin{array}{c}
P_{4}^{\prime}(s) \\
Q_{4}^{\prime}(s)
\end{array}\right]
$$

where the symbols with $<^{\prime}>$ denote the parameters expressed after replacing RSHPA with a pipeline.

Insertion loss (IL) is a key evaluation index to judge the variation conditions at the input of the system load, and it is defined as [16]:

$$
H_{I L}(s)=20 \log \left|\frac{P_{4^{\prime}}(s)}{P_{4}(s)}\right| .
$$

It can be observed that the larger the value of IL is, the better the suppression performance of the RSHPA is. In general, the value of IL is expected to be positive, which means the pulsation to the load is suppressed compared to that without RSHPA. It is notable that IL is not only determined by the performance of the RSHPA, but also affected by the other hydraulic components and pipelines in the hydraulic system. Thus, IL can reflect the performance of RSHPA in a practical fluid power system. 
The frequency characteristics of the pressure ratio (PR) of load to source can be used to evaluate whether the pulsation from the source is amplified at the load position. PR is formulized as:

$$
H_{P R}(s)=20 \log \left|\frac{P_{4}(s)}{P_{1}(s)}\right| .
$$

In contrast to IL, a negative value of PR indicates that the source pulsation is suppressed at the load. The smaller the PR is, the better the source pulsation is suppressed at the load. The frequency characteristics of input flow is determined by the pump. Therefore, the input flow before and after installing the RSHPA stays the same as:

$$
Q_{1}(s)=Q_{1}^{\prime}(s)
$$

The frequency characteristics of load (throttle valve) is assumed to be unchanged, thus [17]:

$$
\frac{P_{4}(s)}{Q_{4}(s)}=\frac{P_{4}^{\prime}(s)}{Q_{4}^{\prime}(s)}=\frac{2 P_{0}}{Q_{0}}
$$

where $P_{0}$ and $Q_{0}$ denote the average pressure and flow at the inlet of the load. Combining Equations (10)-(15), IL and PR can be converted to the following form:

$$
\begin{gathered}
H_{I L}(s)=20 \lg \left|\frac{2 P_{0} a_{21}+a_{22} Q_{0}}{2 P_{0} a_{21}^{\prime}+a_{22}^{\prime} Q_{0}}\right| \\
H_{P R}(s)=20 \log \left|\frac{2 P_{0}}{2 P_{0} a_{11}+a_{12} Q_{0}}\right| .
\end{gathered}
$$

In the later section, Equations (17) and (18) are used to optimize the performance of RSHPA.

\section{Adaptive Particle Swarm Optimization (PSO) Algorithm}

In this section, the conventional particle swarm optimization (PSO) method is introduced firstly, and then an adaptive PSO algorithm (APSO) is provided to improve the convergence speed of the optimization process of RSHPA. In the conventional PSO algorithm [18], a member in the swarm is called a particle, which is possibly the solution of the globally best target. During the iteration process, the particle tunes its search ways to track the globally best position on the basis of an objective function and a velocity. The velocity $v_{i}^{d}$ and position $x_{i}^{d}$ of the $d$-th dimension of the $i$-th particle are updated as:

$$
\begin{gathered}
v_{i}^{d}=v_{i}^{d}+\alpha_{1} \text { rand }_{1}^{d}\left(\text { pbest }_{i}^{d}-x_{i}^{d}\right)+\alpha_{2} \text { rand }_{2}^{d}\left(\text { gbest }^{d}-x_{i}^{d}\right) \\
x_{i}^{d}=x_{i}^{d}+v_{i}^{d}
\end{gathered}
$$

where $\alpha_{1}$ and $\alpha_{2}$ denote the constants reflecting the weight of stochastic acceleration terms that drive each particle toward the personally best $p b e s t_{i}^{d}$ and globally best $g b e s t^{d}$, respectively, rand ${ }_{1}^{d}$ and $r a n d_{2}^{d}$ denote the random numbers drawn from the uniform distribution within $[0,1]$. In this paper, the velocity and the position vectors are expressed as $\mathbf{V}_{i}=\left[v_{i}^{1}, v_{i}^{2}, \ldots, v_{i}^{D}\right]$ and $X_{i}=\left[x_{i}^{1}, x_{i}^{2}, \ldots, x_{i}^{D}\right]$ where $D$ stands for the dimension of the search space.

\subsection{Adaptive PSO Algorithm}

The main objective to develop the APSO algorithm is to adaptively adjust the particle number while searching the globally best solution. The conventional PSO algorithm and most PSO variants usually work with a fixed number of particles. When the particles are distributed in a crowded way in a local region, each particle would have a similar distance to the globally best position. This means that some particles represent the same evolution process and are not necessary at this stage. In addition, 
for some complicated optimization problems, researchers generally prefer strong global search ability to allocate particles to a broader search space. However, this strategy lowers the computational efficiency for most unrotated problems [19].

To improve the computational efficiency of the optimization process for RSHPA, APSO would reduce the particle close to the global best. First, a distance factor $\delta_{i}$ is given as:

$$
\delta_{i}=\frac{f\left(X_{i}\right)-f_{\min }}{f_{\max }-f_{\min }}, i=1,2, \ldots, N
$$

where $f(\cdot)$ denotes the objective function value (OFV), $N$ denotes the total number of particles, $f_{\max }$ denotes the OFV regarding the global best, and $f_{\min }$ denotes the smallest OFV during all the calculation results of particles. The larger the distance factor, the closer the distance to the global best. In APSO, the particle removal is only conducted at the convergence stage, which satisfies:

$$
\sum_{k=i}^{i+W}\left|f_{\max }^{k}-f_{\max }^{k-1}\right|=0
$$

where $k$ denotes the iteration order and $W$ denotes the consecutive times for the condition that the best OFV keeps unchanged. One should note that the number of particles has a lower bound $N_{\min }$. Based on Equations (18)-(21), the flow chart of APSO is summarized as Figure 4 where the green parts denote the iteration steps in the conventional PSO algorithm, and the yellow parts denote the improved content in the APSO algorithm. The initialization step includes assigning initial position and velocity, personally best particle and globally best particle. During searching, each particle has position limitation, which represents the geometry limitation for the size variables of RSHPA in the optimization design. The velocity also has limitations and it affects the search speed of the APSO algorithm. Although a small limitation range can improve the optimization accuracy of each geometry parameters, the fabrication precision of RSHPA cannot reach too high in a practical structure. Thus, the velocity is set to a value close to the fabrication tolerance in this paper. Note that the unit of velocity in the optimization problem is equal to the unit of the size variables. 


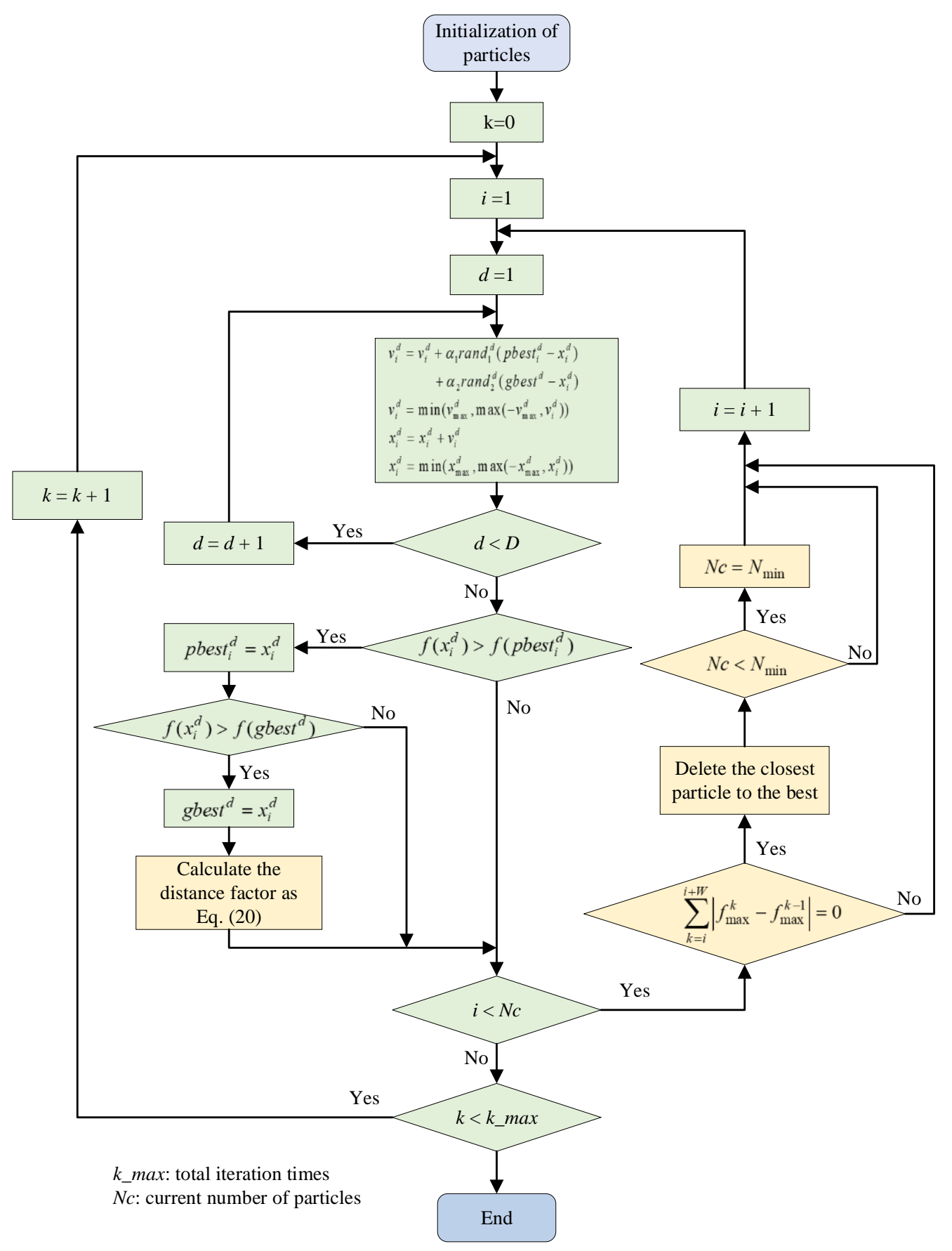

Figure 4. Flow chart of the adaptive particle swarm optimization (APSO) algorithm.

\subsection{Numerical Test of Adaptive Particle Swarm Optimization (APSO)}

The APSO algorithm is tested using two benchmark functions and compared with the conventional PSO (CPSO) algorithm and an improved PSO (IPSO) algorithm proposed in [20]. The IPSO was proposed for identifying the model parameters with different order of magnitudes. The geometry size of the expansion chamber and strings absolutely have a different order of magnitude. First, two benchmark functions are given as follows [19]: 
A. Six-hump camel-back function

$$
F_{1}(x)=\sum_{i=1}^{D}\left(x_{i} \sin \left(\sqrt{\left|x_{i}\right|}\right)\right)
$$

B. Ackley function

$$
F_{2}(x)=-20 \exp \left(-0.2 \sqrt{\frac{1}{10} \sum_{i=1}^{2} x_{i}^{2}}\right)-\exp \left(\frac{1}{10} \sqrt{\sum_{i=1}^{2} \cos \left(2 \pi x_{i}\right)}\right)+20+e .
$$

Schwefel and Ackley functions have one narrow global optimum and many shallow local optima. The global optimum of two benchmark functions and its corresponding variable values are given in Table 1. Note that the global optimum of two functions are both minimal values of the functions under given search ranges.

Table 1. Fundamental information of the benchmark functions.

\begin{tabular}{ccccc}
\hline $\boldsymbol{F}$ & Name & $\boldsymbol{X}^{*}$ & $\boldsymbol{F}\left(\boldsymbol{X}^{*}\right)$ & Search Range \\
\hline$F_{1}$ & $\begin{array}{c}\text { Six-Hump } \\
\text { Camel-Back }\end{array}$ & {$[-0.0898,0.7126]$} & -1.0316 & {$[-50,50]$} \\
\hline$F_{2}$ & Ackley & {$[0,0]$} & 0 & {$[-50,50]$} \\
\hline
\end{tabular}

Table 2 demonstrates the fundamental parameters used in APSO. The constant $c_{1}$ and $c_{2}$ are chosen to be 2, which proves to have performed well in many works in the literature [21]. The number of particles, the lower bound of particle number and the parameter $W$ are empirically determined according to our repeated tests, which aim to improve the computational efficiency and simultaneously keep acceptable solution accuracy.

Table 2. Fundamental parameters for APSO.

\begin{tabular}{cccccc}
\hline Parameter & $c_{1}$ & $c_{2}$ & $W$ & $N$ & $N_{\text {min }}$ \\
\hline Value & 2 & 2 & 3 & 20 & 10 \\
\hline
\end{tabular}

Figure 5a demonstrates the variation process of OFV of the Six-Hump Camel-Back function. It is observed that although the conventional PSO algorithm has the best evolution speed at the most steps of iteration, APSO can still catch up with the OFV of CPSO when the iteration step is near 200. This result indicates that even if the number of particles is reduced, it would not affect the search ability of the APSO algorithm obviously. The search speed of the IPSO algorithm is lower than both CPSO and APSO because the algorithm focuses on global search ability to avoid missing the possible solution in complicated optimization problems. However, for common geometry parameter optimization, its computational efficiency is not satisfactory. The test results using the Ackley function (Figure 5b) are similar to that of Six-Hump Camel-Back function.

Table 3 shows the average function calls for 10 repeated runs. Here, the function call refers to the process to analyze each particle's OFV during iteration. The test result shows that the APSO algorithm uses the least function calls in the benchmark function-based tests. More specifically, the APSO algorithm reduces the function calls by $20.6 \%$ and $65.0 \%$ compared to CPSO and IPSO, respectively, when using Six-Hump Camel-Back function. In the test using the Ackley function, the APSO algorithm reduces the function calls by $14.3 \%$ and $27.6 \%$ compared to the CPSO and IPSO algorithms. These results satisfy the OFV results given in Figure 5 and also meet the design objective of APSO. 


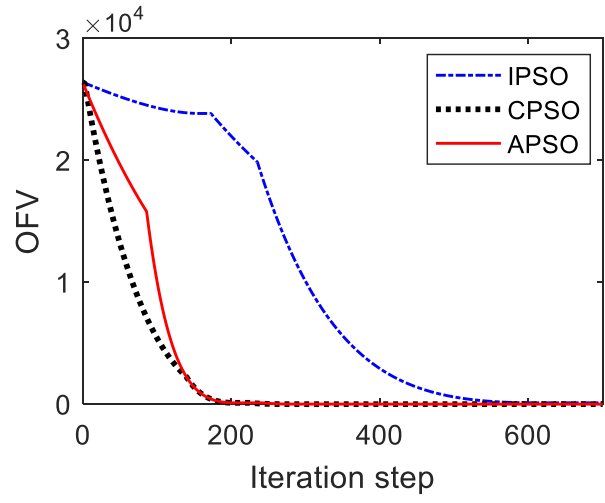

(a) Six-hump camel-back function

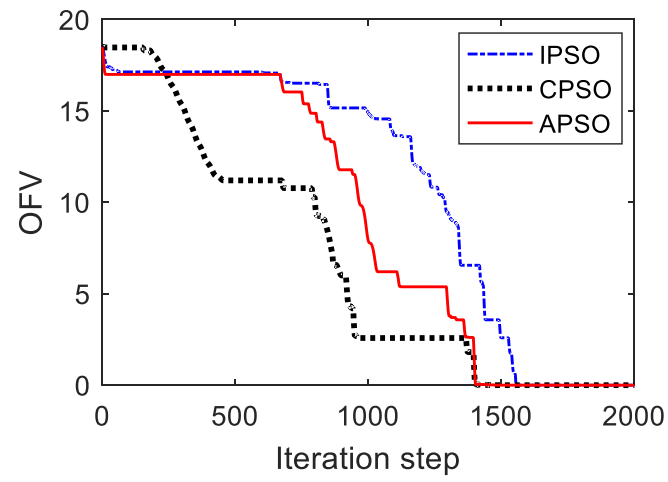

(b) Ackley function

Figure 5. Objective function value (OFV) variation process of two benchmark functions.

Table 3. Function calls comparison.

\begin{tabular}{cccc}
\hline Average Function Calls & CPSO & APSO & IPSO \\
\hline$F_{1}$ (Six-Hump Camel-Back function) & 4018 & 3190 & 9125 \\
$F_{2}$ (Ackley function) & 27,124 & 23,220 & 32,050 \\
\hline
\end{tabular}

\section{Optimization of RSHPA}

The optimization objective is to enhance the performance of RSHPA in wide band and specific frequency conditions, respectively. Thus, we analyze the performance of different types of RSHPA and determine their appropriate usage scenarios. As introduced before, IL and PR are two evaluation indexes of RSHPA. Maximizing IL and minimizing PR both can improve the attenuation performance of RSHPA effectively.

In a practical fluid power system, RSHPA has geometry restrictions because of the increased fabrication cost, installation difficulties and maintenance challenges caused by an inappropriate size of RSHPA. Additionally, the force applied to strings cannot exceed the ultimate strength to prevent the breakage of strings. The following optimization process would consider all the aforementioned restrictions.

\subsection{Geometry Optimization}

Figure 6 demonstrates the schematic diagram of the RSHPA with the symbols of geometry parameters needed to be optimized. It is assumed that the length of the strings is equal to the length of the expansion chamber. Thus, the total variable parameters can be summarized as a vector of $\left(l, D, g, \Theta_{i}\right)^{T}$. As investigated in our previous work [10], the string-fluid resonator only works for specific frequency; as a result, we only optimize the parameters of the expansion chamber in the wide band case, and optimize the parameters of both string-fluid resonators and expansion chamber in the specific frequency case.

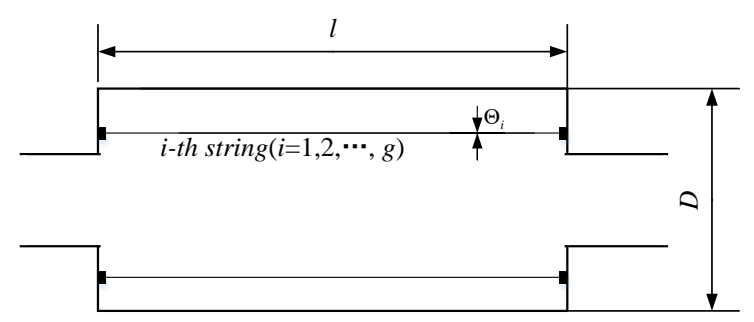

Figure 6. Schematic diagram of the single-chamber RSHPA. 


\subsubsection{Wide-Band Optimization}

As mentioned in the literature [2], the frequency of most hydraulic pulsation is less than $1 \mathrm{kHz}$; thus, this frequency band is selected as the optimization objective in this paper. To optimize the wide-band performance, we construct two objective functions using the sum of uniformly-spaced frequency attenuation effects, and they are formulized as:

$$
\begin{gathered}
O F_{1}(l, D)=-\sum_{i=1}^{1000} I L_{i} \\
O F_{2}(l, D)=-\sum_{i=1}^{1000}\left(k_{1} \frac{I L_{i}-I L_{\min }}{I L_{\max }-I L_{\min }}+k_{2} \frac{P R_{\max }-P R_{i}}{P R_{\max }-P R_{\min }}\right), k_{1}+k_{2}=1
\end{gathered}
$$

where $I L_{i}$ and $P R_{i}$ denote the $I L$ and $P R$ values when the frequency is $i$. The subscripts max and min denote the corresponding maximum and minimum values during iteration. It is notable that the attenuation effects judged by $I L$ and $P R$ are normalized in Equation (25), in order to adjust their contributions in the objective function via two constants $k_{1}$ and $k_{2}$. In addition, two objective functions are both minimization problems.

Figure 7 demonstrates the comparison result using $O F_{1}$ and the related parameter information is given in Table 4 where $g$ denotes the total number of strings. The original values given in the table are the same as the parameter values of the existing RSHPA in our lab. It is observed that the optimized $I L$ is nearly larger than the original results during the entire given frequency band. This result agrees with the optimization objective. Additionally, although the objective function only contains the items of $I L$, the performance of $P R$ is also improved. Thus, improving the performance of $I L$ for wide band conditions can be a simple way to improve the performance of $P R$ in the same condition. One should note that the optimized values of the length and diameter of the RSHPA reach the upper bound of their search ranges, indicating that the larger volume of the RSHPA is, the better the performance for wide band conditions. However, the geometry of RSHPA will be definitely restricted in a practical fluid power system due to the factors of limited installation space, cost, etc. The other phenomenon to note is that the attenuation performance from the string-fluid resonators (see narrow-band peaks) is somewhat reduced and even becomes worse after optimization. This result reveals that whether the string-fluid resonator can well work not only depends on its performance but is also affected by the expansion chamber. Therefore, we can reasonably infer that if a hydraulic system needs to suppress the noise at specific frequency using RSHPA, the wide band optimization is not suitable.

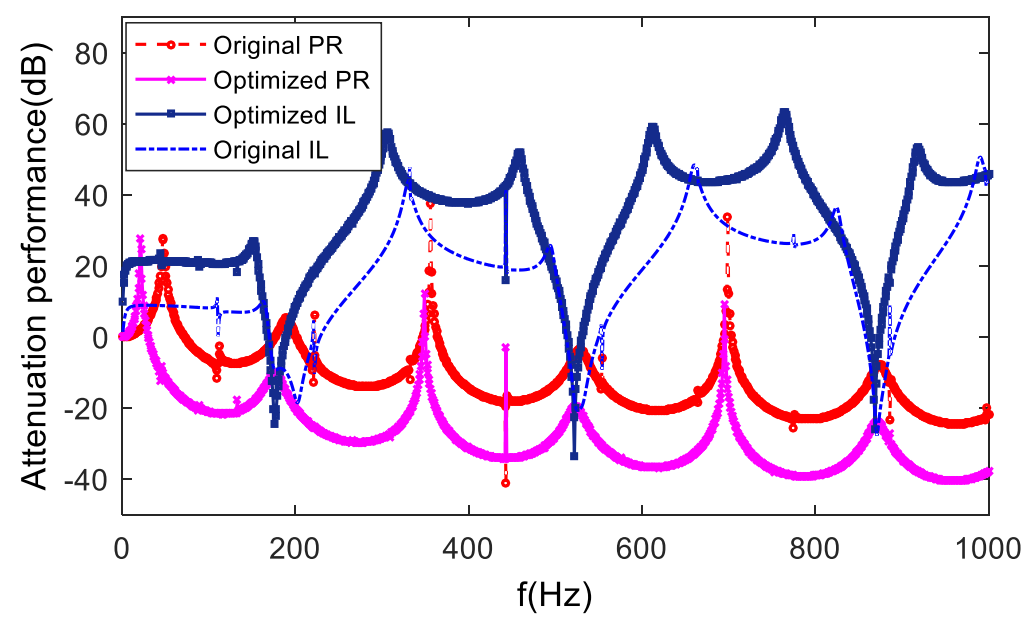

Figure 7. Attenuation performance of single-chamber RSHPA in $O F_{1}$. 
The last row of Table 4 provides the running time of solving the optimization problem using three PSO-based algorithms. Three algorithms all finally converge to a close result. The proposed APSO consumes the least time and reduces time by $23.5 \%$ and $33.4 \%$ compared to CPSO and IPSO, respectively. This numerical result validates the superiority of APSO in computational efficiency. To validate whether the result is globally optimal, we use the traversal search method to calculate again and obtain the same parameters. This result indicates that the global search ability of the APSO algorithm is not weakened in the optimization problem given in this paper. In the rest of this study, the optimized results are all examined by the traversal search method.

As introduced in Section 2.2, a larger value of $I L$ and a smaller value of $P R$ are beneficial to a hydraulic silencer, especially when $I L$ is larger than 0 and $P R$ is smaller than 0 . According to our repeated tests, the optimized values using different combinations of $k_{1}(0.1,0.3,0.5,0.7$ and 0.9$)$ and $k_{2}$ are all close to the results computed using $O F_{1}$. Thus, it may be concluded that the objective function only containing $I L$ items or both $I L$ and $P R$ items will lead to a same result.

Table 4. Parameter information used in Figure 7.

\begin{tabular}{ccccccc}
\hline Parameter & $\boldsymbol{l}(\mathbf{m})$ & $\boldsymbol{D}(\mathbf{m})$ & $g$ & $\boldsymbol{\Theta}_{\boldsymbol{i}}(\mathbf{m m})$ & $\boldsymbol{\rho}_{\mathbf{0}}\left(\mathbf{k g} / \mathbf{m}^{\mathbf{3}}\right)$ & $\boldsymbol{\mu}_{\mathbf{0}}(\mathbf{P a} \cdot \mathbf{s})$ \\
\hline Search range & {$[0.2,0.35]$} & {$[0.05,0.15]$} & {$[1,30]$} & {$[0.1,0.4]$} & 890 & 0.034 \\
\hline Original value & 0.25 & 0.08 & 5 & 0.4 & 890 & 0.0344 \\
\hline Optimized value & 0.35 & 0.15 & APSO: 610.3s CPSO: 797.8s IPSO: 916.0s \\
\hline Running time & \multicolumn{7}{c}{} \\
\hline
\end{tabular}

\subsubsection{Specific Frequency Optimization}

To enhance the performance of RSHPA at specific frequency, the natural frequency of the strings should be tuned to the corresponding frequency. Here, $220 \mathrm{~Hz}$ is selected as the optimized goal. The search ranges, original values and optimized values calculated using the APSO algorithm are given in Table 5 where $T_{i}$ and $\Theta_{i}$ denotes the applied tension and diameter of the $i$-th string. The symbol $\left[\sigma_{S A F E}\right]$ denotes the allowable stress of the strings. It can be observed that APSO is still the most computationally efficient between three algorithms, and reduces the running time by $22.2 \%$ and $37.1 \%$ compared to that of CPSO and IPSO algorithms.

Table 5. Parameter information used in Figure 8.

\begin{tabular}{cccccc}
\hline Parameter & $\boldsymbol{l}(\mathbf{m})$ & $\boldsymbol{D}(\mathbf{m})$ & $g$ & $\boldsymbol{\Theta}_{\boldsymbol{i}}(\mathbf{m m})$ & $\boldsymbol{T}_{\boldsymbol{i}}(\mathbf{N})$ \\
\hline Search range & {$[0.2,0.35]$} & {$[0.05,0.15]$} & {$[1,30]$} & {$[0.1,0.4]$} & {$[1,50]$} \\
\hline Constraint condition & \multicolumn{5}{c}{$T_{i} /\left[\pi\left(\Theta_{i} / 2\right)^{2}\right]<\left[\sigma_{S A F E}\right]$} \\
\hline Original value & 0.25 & 0.088 & 1 & 0.2 & 1.8 \\
\hline Optimized value & 0.35 & 0.15 & 10 & 0.4 & 7.7 \\
\hline Running time & \multicolumn{7}{c}{ APSO: 11.2 s CPSO: 14.4 s IPSO: $17.8 \mathrm{~s}$} \\
\hline
\end{tabular}




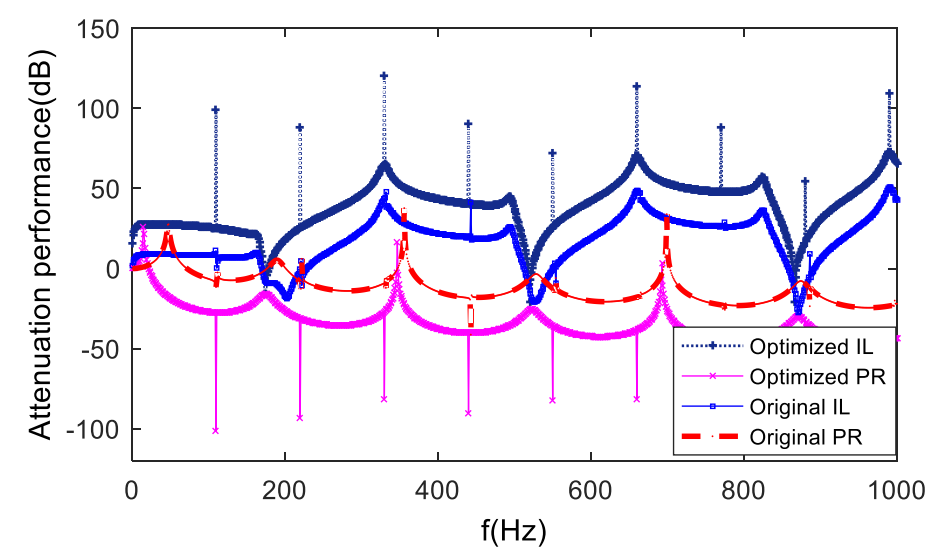

Figure 8. Attenuation performance of single-chamber RSHPA in $\mathrm{OF}_{3}$.

The natural resonant frequency of strings is formulized as [22]:

$$
f_{\text {in }}=\frac{n}{2 l} \sqrt{\frac{T_{i}}{\delta}}
$$

where $\delta$ denotes the linear density of the strings. In this paper, $\delta$ is calculated by $\delta=\rho \pi \Theta^{2} / 4$ where $\rho$ denotes the bulk density and it is determined by the material of the commercial string. In this paper, $\rho$ is $8110 \mathrm{~kg} / \mathrm{m}^{3}$. Parameter $f_{\text {in }}$ denotes the $n$-th order frequency response of the $i$-th string. In general, $f_{\text {in }}$ is equal to the pulsation frequency of the noise source, which is the axial piston pump in this paper. The pulsation frequency is calculated by [10]:

$$
f_{\text {pul }}=\frac{n_{p} Z}{60}
$$

where $n_{p}$ denotes the rotational speed of the motor and $Z$ denotes the piston number of the pump.

It is assumed that all strings are consistent. The objective function is given as follows:

$$
O F_{3}\left(l, D, g, \Theta_{i}, T_{i}\right)=-I L_{220}
$$

Figure 8 demonstrates the comparison results using the original and optimized values in the specific frequency optimization case. It is observed that the attenuation performance at the desired 220 $\mathrm{Hz}$ is enhanced obviously, which corresponds to the second-order response of the strings. Additionally, the other order responses of the strings are also improved, indicating that the enhancement is useful for all responses even if we only optimize one of them. Note that the effective frequency band for enhancement of the desired frequency is narrow. Thus, the fabrication error and parameter drift in a practical device would easily make the characteristics of the RSHPA deviate from the design objective.

Two methods can be used to solve the above problem. One takes advantage of the peaks generated by the expansion chamber, which has a wide effective band, such as the effect at $490 \mathrm{~Hz}$ in Figure 8 . However, the peaks caused by the strings and the expansion chamber are difficult to both maximize at any specified frequency. It can be observed in Figure 9 that the curve in magenta, which is obtained by tuning the peaks generated by the expansion chamber (without tuning the parameters of strings), has the same peak frequency as the other cases. However, its resonant frequency from strings is different from the peak position generated by the expansion chamber. Although tuning the peaks position can improve the performance at desired frequency, the valleys of IL would possibly increase (one more in Figure 9). The other method is extending the effective bandwidth by deploying different strings with close resonant frequencies. Around $220 \mathrm{~Hz}, 10$ strings with different uniformly-spaced resonant frequency are deployed and thus present an abrupt change in graph (the performance from 
the expansion chamber is not maximized here). The effective frequency band at each desired frequency is obviously extended compared to that using single-frequency strings (deep blue dotted curve).

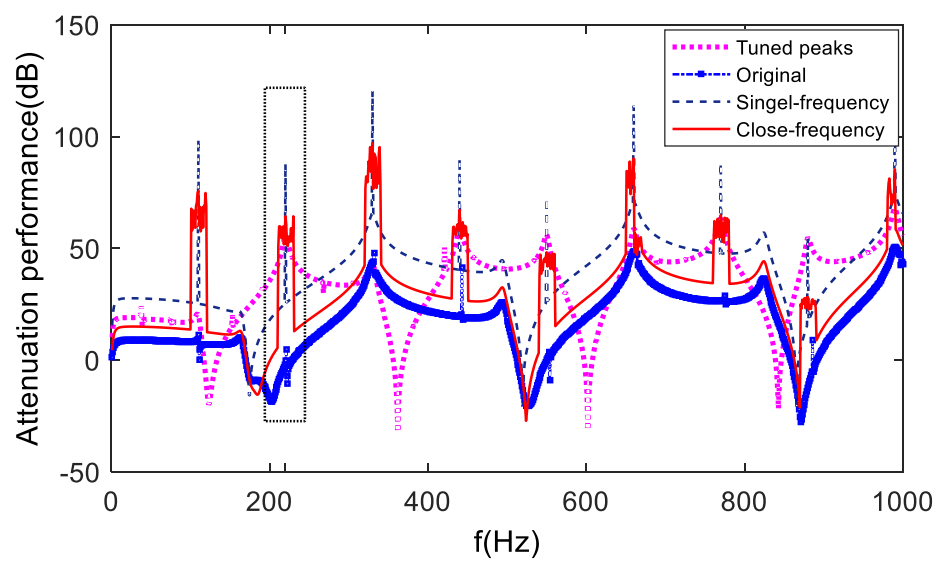

Figure 9. Attenuation effects after extending the performance around $220 \mathrm{~Hz}$.

\subsubsection{Experiment}

To validate the proposed method, we designed and fabricated the test platform of RSHPA, as shown in Figure 10a. Figure 10b demonstrates the inner structure of the RSHPA. The schematic diagram of the test platform is shown in Figure 2 where the pressure sensors are mounted before and after the RSHPA, respectively. The accuracy of the pressure sensors is $\pm 0.25 \%$ and the maximum frequency that can be measured is $1 \mathrm{kHz}$. The pulsation source is generated by a plunger pump with 9 plungers. The rated pressure, flow and power of the pump are $25 \mathrm{MPa}, 180 \mathrm{~L} / \mathrm{min}$ and $93 \mathrm{~kW}$, respectively. The length of upstream and downstream pipes is $2.11 \mathrm{~m}$ and $3.41 \mathrm{~m}$, respectively. The diameter of the expansion chamber and pipes is $0.042 \mathrm{~m}$ and $0.15 \mathrm{~m}$, respectively. The effective length of the expansion chamber and strings is both $0.35 \mathrm{~m}$. The load in the test is a throttle valve, whose working flow is between the range of $10 \mathrm{~L} 200 \mathrm{~L} / \mathrm{min}$.

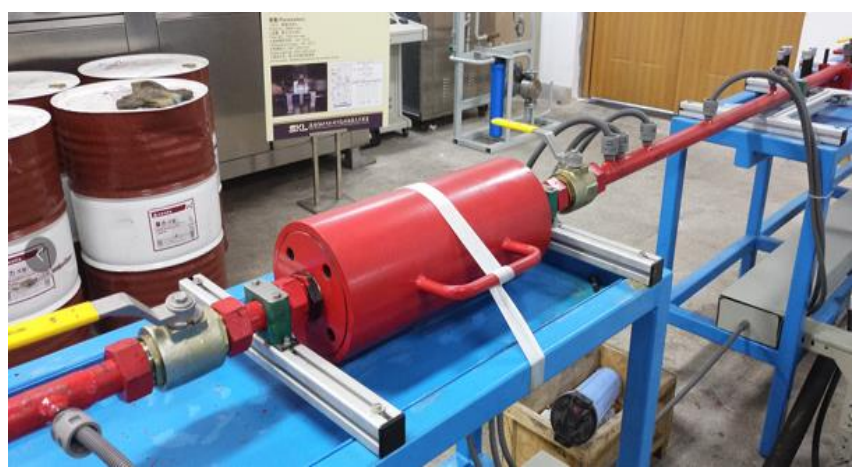

(a) Test platform

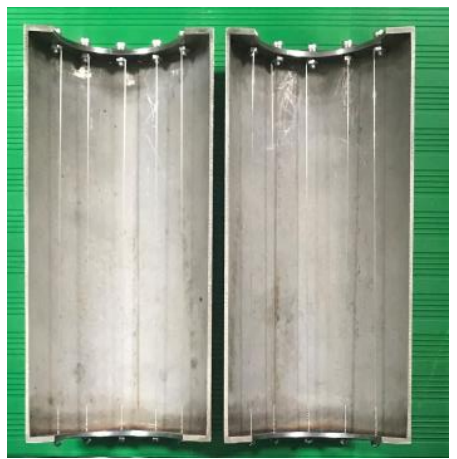

(b) Inner structure

Figure 10. Experiment platform for testing the RSHPA.

Figure 11 demonstrates the comparison results between the theoretical curve and experiment data. It can be seen that when RSHPA only contains single string, IL in $110 \mathrm{~Hz}$ and $220 \mathrm{~Hz}$ does not present expected attenuation strength. This is because the effective attenuation frequency band of the string is narrow and when there is some error in parameter values or fabrication, the string-fluid resonator cannot attenuate noise at the desired frequency. In contrast, the multi-string combination shows significant attenuation effect at the design frequency $(220 \mathrm{~Hz})$ and the other order response frequencies of the string. It proves that the multi-string combination can effectively broaden the attenuation band of the target frequency, improving the system tolerance for machining and value selection. 


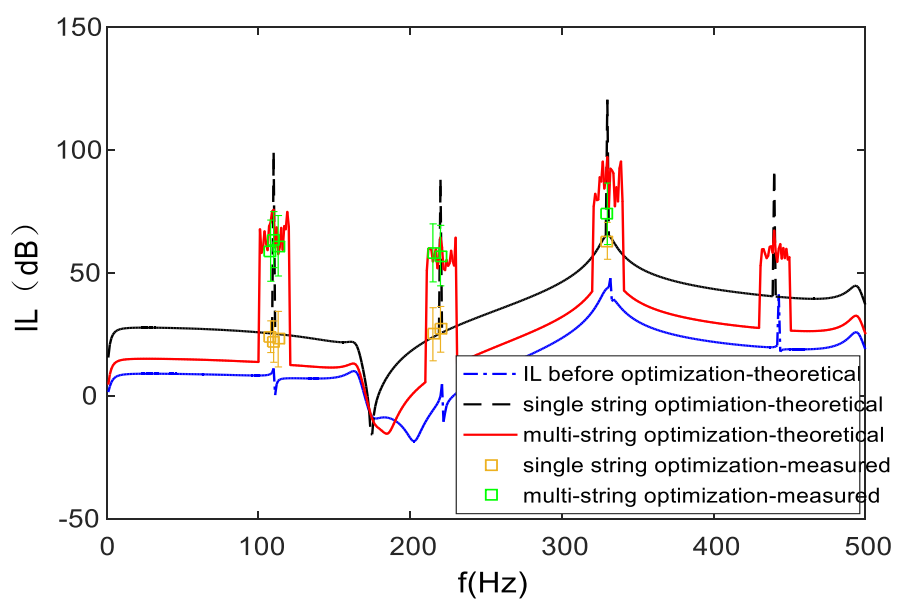

Figure 11. Comparison of the theoretical and experimental results.

\subsection{Configuration Optimization}

To investigate the potential configuration for improving the performance of RSHPA, serial and parallel combinations of RSHPA were tested and optimized, and their schematic diagrams are given in Figure 12. For serial configuration, the length and diameter of each chamber was kept the same. For parallel configuration, the length (diameter) of each RSHPA was also assumed to be consistent. To better compare multiple-chamber configuration with the single-chamber, the maximum total length and width of the combination configuration were equal to the upper bound in optimization of the single-chamber case, i.e., the occupied physical space of the multiple-chamber RSHPA could not exceed the single-chamber configuration. It was assumed that the length of the connection pipelines for each chamber was short enough to be ignored.

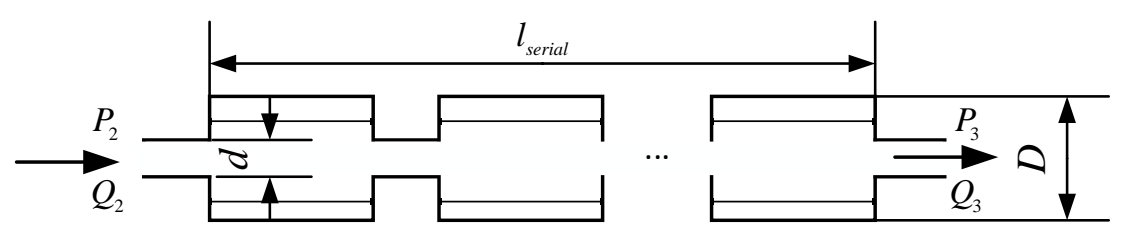

(a) Serial RSHPA

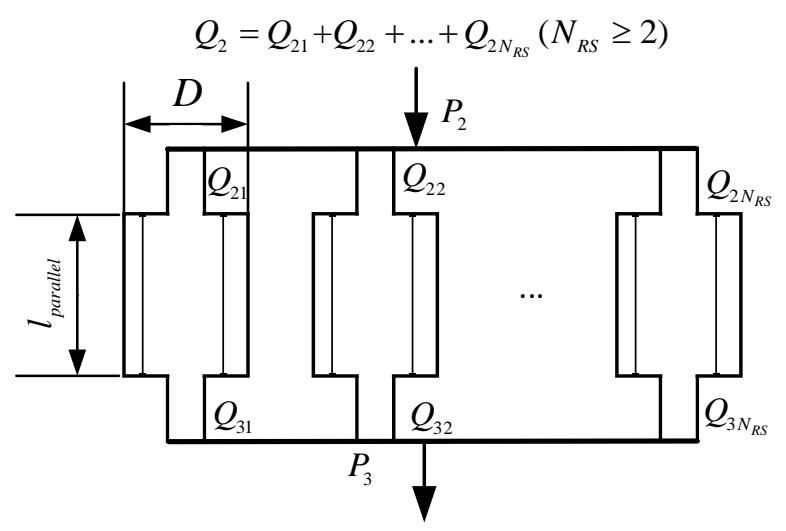

(b) Parallel RSHPA

Figure 12. Schematic diagram of serial and parallel combinations of RSHPA. 
The transfer matrix of two multiple-chamber RSHPA are different from the single-chamber form and the serial RSHPA is formulized as:

$$
\mathbf{A}_{\text {serial }}=\prod_{i=1}^{N_{R S}} \mathbf{A}_{2}^{i}=\prod_{i=1}^{N_{R S}}\left[\begin{array}{ll}
a_{11}^{i} & a_{12}^{i} \\
a_{21}^{i} & a_{22}^{i}
\end{array}\right]
$$

where $N_{R S}$ denotes the number of RSHPA, $\mathbf{A}_{2}^{i}=\left[a_{11}^{i}, a_{12}^{i} ; a_{21}^{i}, a_{22}^{i}\right]$ denotes the transfer matrix of $i$-th RSHPA, and $\mathbf{A}_{\text {serial }}$ denotes the total transfer matrix of the serial RSHPA. Because the flow going through each RSHPA is the same, the total transfer matrix of parallel RSHPA can be derived as:

$$
\mathbf{A}_{\text {parallel }}=\left[\begin{array}{cc}
a_{11}^{i} & a_{12}^{i} / N_{R S} \\
N_{R S} a_{21}^{i} & a_{22}^{i}
\end{array}\right] .
$$

\subsubsection{Wide-Band Optimization}

Table 6 demonstrates the parameter information used for multiple-chamber wide-band optimization cases. Figure 11 shows the optimization results for both serial and parallel RSHPA. It can be observed that the optimized results of serial RSHPA are similar to that of single-chamber cases, while the performance of optimized parallel RSHPA is apparently worse than the other two devices. It may be concluded that the single-chamber RSHPA is highly competitive to multiple-chamber RSHPA in wide-band optimization problems. In addition, the attenuation effects from the strings are not obvious. This is because maximization of the performance at specific frequency contributes little to wide-band performance enhancement, and the optimized geometry parameters would converge to the result given in Figure 13.

Table 6. Parameter information for multiple-chamber in wide-band optimization.

\begin{tabular}{cccccccc}
\hline Parameter & $\boldsymbol{l}_{\text {serial }}(\mathbf{m})$ & $\boldsymbol{l}_{\text {parallel }}(\mathbf{m})$ & $\boldsymbol{D}(\mathbf{m})$ & $g$ & $\boldsymbol{N}_{\boldsymbol{R} S}$ & $\boldsymbol{\Theta}(\mathbf{m m})$ & $\boldsymbol{T}_{\boldsymbol{i}}(\mathbf{N})$ \\
\hline Search range & {$[0.2,0.35]$} & {$[0.2,0.35]$} & {$[0.2,0.35]$} & {$[1,30]$} & {$[2,5]$} & {$[0.1,0.4]$} & {$[10,300]$} \\
\hline Constraint condition & & $T_{i} /\left[\pi\left(\Theta_{i} / 2\right)^{2}\right]$ & $<\left[\sigma_{S A F E}\right], N_{R S} D \leq 0.15$ & (for the parallel case) & \\
\hline Original value & 0.2 & 0.2 & 0.08 & 5 & 2 & 0.2 & 50 \\
\hline Serial optimization & 0.35 & & 0.15 & 1 & 2 & 0.4 & 7.7 \\
\hline Parallel optimization & & 0.35 & 0.15 & 30 & 2 & 0.4 & 7.7 \\
\hline
\end{tabular}

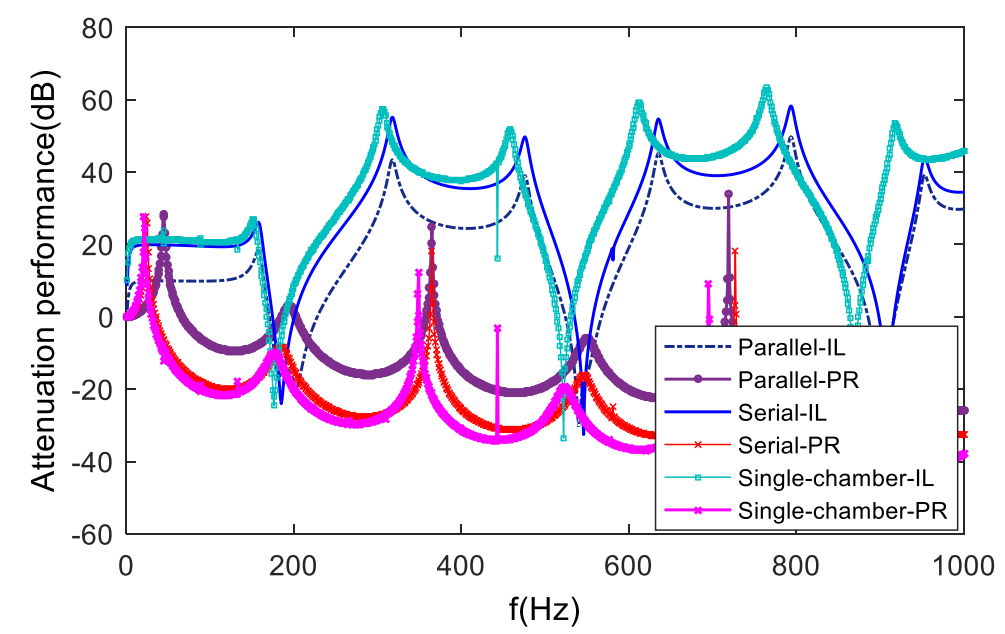

Figure 13. Wide-band optimization for serial and parallel combinations of RSHPA. 


\subsubsection{Specific Frequency Optimization}

Table 7 shows the parameter information of the multiple-chamber RSHPA for optimizing the performance at $220 \mathrm{~Hz}$ and Figure 14 shows the corresponding comparison results between serial, parallel and single-chamber RSHPA. It can be observed that the single-chamber RSHPA has the best performance at $220 \mathrm{~Hz}$, as well as the other responding frequency of strings. One should note that the IL curve of parallel RSHPA has an oscillation near $220 \mathrm{~Hz}$. Although parallel configuration can improve the performance at desired frequency, negative effects arise at nearby frequency. This condition should be avoided. PR curves have similar results compared to that of $I L$ where the single-chamber configuration has the best performance.

Because the numerical model of RSHPA was validated in our previous work and the above optimization results (Table 7) were also verified by the traversal method, it may be concluded that the single-chamber RSHPA has better performance under the same volume compared to serial and parallel configurations.

Table 7. Parameter information for multiple-chamber in specific frequency optimization.

\begin{tabular}{cccccccc}
\hline Parameter & $l_{\text {serial }}(\mathbf{m})$ & $l_{\text {parallel }}(\mathbf{m})$ & $\boldsymbol{D}(\mathbf{m})$ & $g$ & $\boldsymbol{N}_{R S}$ & $\boldsymbol{\Theta}(\mathbf{m m})$ & $\boldsymbol{T}_{\boldsymbol{i}}(\mathbf{N})$ \\
\hline Search range & {$[0.2,0.35]$} & {$[0.2,0.5]$} & {$[0.08,0.15]$} & {$[1,30]$} & {$[2,5]$} & {$[0.1,0.4]$} & {$[1,50]$} \\
\hline Constraint condition & & $T_{i} /\left[\pi\left(\Theta_{i} / 2\right)^{2}\right]$ & $<\left[\sigma_{S A F E}\right], N_{R S} D \leq 0.15$ (for the parallel case) \\
\hline Original value & 0.2 & 0.2 & 0.08 & 5 & 2 & 0.2 & 50 \\
\hline Serial optimization & 0.32 & & 0.15 & 1 & 5 & 0.1 & 0.52 \\
\hline Parallel optimization & & 0.35 & 0.15 & 1 & 5 & 0.4 & 7.7 \\
\hline
\end{tabular}

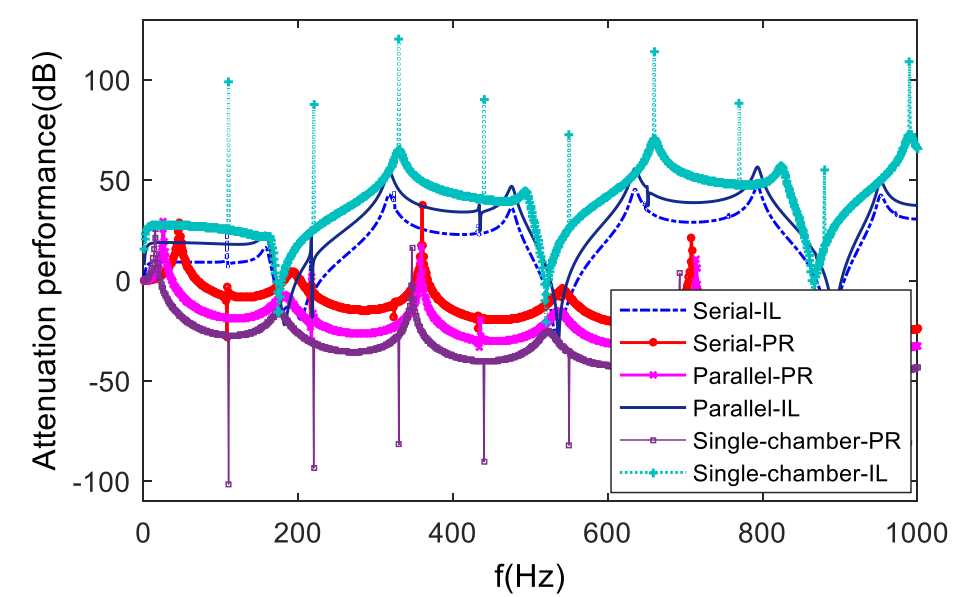

Figure 14. Specific frequency optimization for serial and parallel combinations of RSHPA (220 Hz).

\subsection{Installation Position Optimization}

This section aims to find the most suitable installation location of the RSHPA in a straight pipe line system, so as to achieve the optimal attenuation effect of the RSHPA. The optimization goal is still $220 \mathrm{~Hz}$ and the geometrical and physical parameters in the optimization process remain the same as before. The only variable is the position of the RSHPA in the straight pipeline system.

As mentioned, the effective frequency band can be extended by deploying multiple strings with uniformly-spaced resonant frequency. In addition, the resonant frequency of the RSHPA can also be identified by experiments. The identification steps can be summarized as: (1) Tune the rotational speed of the driving motor of the pump to the designed points. The pulsation frequency is calculated by Equation (26). (2) Transform the pressure data at the outlet of the RSHPA into the frequency domain by fast Fourier transform (FFT) and observe the power spectrum in real-time. (3) Fine tune the rotational 
speed of the motor until the power spectrum captures an obvious decrease at the peaks. (4) Inversely estimate the tension on the string and finely tune the tension until the pulsation can be attenuated at the designed operating frequency.

Figure 15 shows the comparison results of the theoretical curve and the measured data with the uncertainty represented by the error bar. The average deviation in attenuation strength is of $22.6 \mathrm{~dB}$. The horizontal coordinate denotes the distance between the pump and the RSHPA. Through calculation, it is found that when the RSHPA is installed at the inlet, the middle of the pipeline or near the load, the insertion loss of the RSHPA at $220 \mathrm{~Hz}$ can both reach the maximum value. In the experiment, some positions were selected for insertion loss measuring and the measured data are given with the error bar. The test results show that the measured values are basically consistent with the theoretical values.

In order to further explore the universality of RSHPA layout, $150 \mathrm{~Hz}$ and $390 \mathrm{~Hz}$ are selected as the optimization objectives. Figure 16 shows the influence of RSHPA layout on insertion loss at $150 \mathrm{~Hz}$ and $390 \mathrm{~Hz}$. The uncertainty in attenuation strength for them is $17.2 \mathrm{~dB}$ and $21.1 \mathrm{~dB}$, respectively. It can be seen that although RSHPA has extreme value points in the middle of the pipeline, not all of them are maxima or even minima. Therefore, optimizing IL at different frequency would have different optimal position and each case should be analyzed by the numerical model using the optimization algorithm.

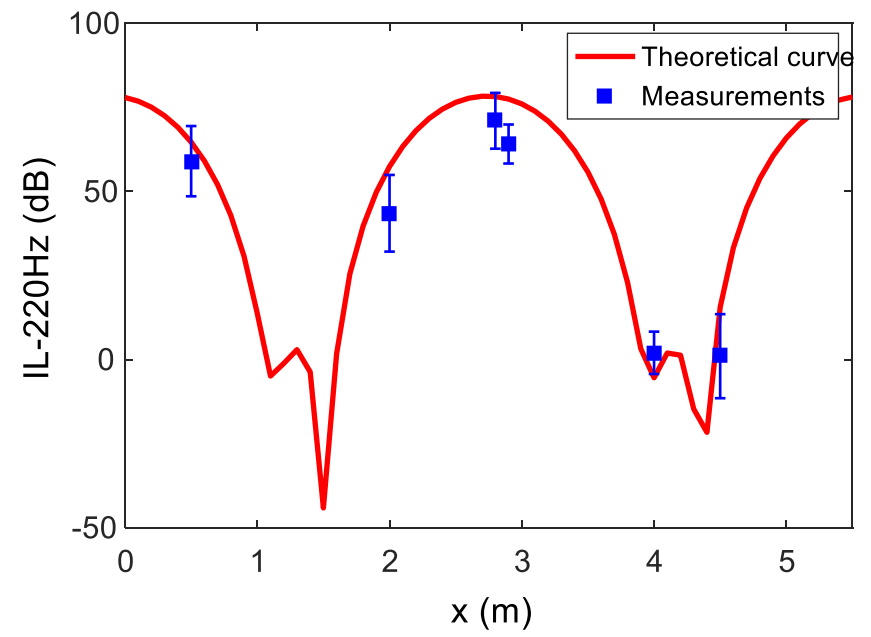

Figure 15. Optimization of installation position of RSHPA $(220 \mathrm{~Hz})$.
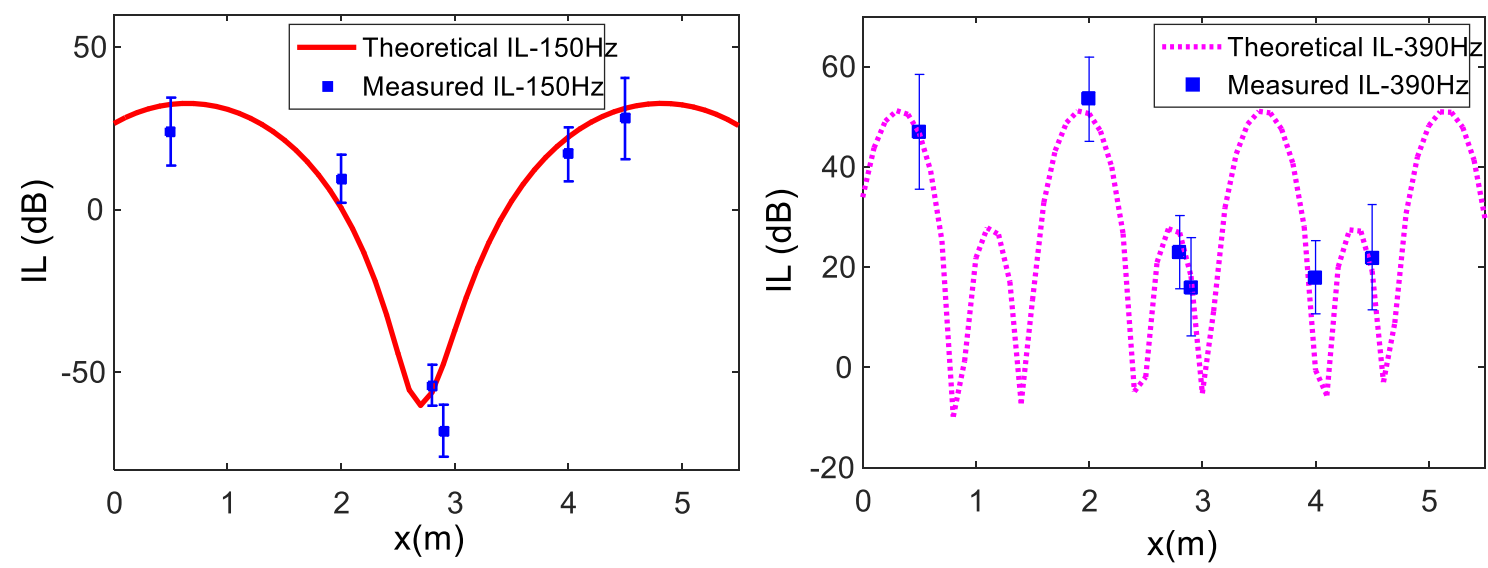

Figure 16. Optimization of installation position of RSHPA $(150 \mathrm{~Hz}$ and $390 \mathrm{~Hz})$. 


\section{Conclusions}

This paper demonstrates the model optimization method for a resonant strings-based hydraulic pulsation attenuator. By contrast with a conventional study, which only considers the geometry optimization, improving the optimization computational efficiency, investigating potential improved configuration and installation position are also discussed in this study. The main contributions of this paper are summarized as:

(1) An adaptive particle swarm optimization (APSO) algorithm is proposed to improve the computational efficiency for RSHPA optimization. The APSO algorithm proves to be better in computational efficiency compared to CPSO and IPSO algorithms in benchmark function tests and the RSHPA optimization problem.

(2) To extend the narrow effective bandwidth at the desired frequency, deploying close natural frequency strings and tuning the peak position of RSHPA are investigated, both of which can extend the effective bandwidth at desired positions.

(3) The serial and parallel configuration of the RSHPA are investigated. The test result proves that the single-chamber RSHPA has better attenuation performance at desired frequency. In addition, the effects caused by the installation position of the RSHPA in a pipeline system are studied. The test result indicates that different optimization frequency corresponds to a different optimal installation position.

Author Contributions: X.S. wrote the manuscript, H.Z. and H.Y. reviewed the manuscript and provided some modification suggestions. All authors have read and agreed to the published version of the manuscript.

Funding: This work was supported in part by the National Natural Science Foundation of China under grant 51890880 and 51890885, and in part by the National Key Research and Development Project under grant 2018YFB2001203.

Conflicts of Interest: The authors declare no conflict of interest.

\section{References}

1. Kela, L. Resonant frequency of an adjustable Helmholtz resonator in a hydraulic system. Arch. Appl. Mech. 2009, 79, 1115-1125. [CrossRef]

2. Zhu, J.; Zhou, H.; Yang, H. Investigation of the pulsation-reduction characteristics of an expansion chamber attenuator in a hydraulic system. Proc. Inst. Mech. Eng. Part C J. Mech. Eng. Sci. 2017, 232, 872-880. [CrossRef]

3. Huang, H.; Chen, Z.; Ji, Z. One-way fluid-to-acoustic coupling approach for acoustic attenuation predictions of perforated silencers with non-uniform flow. Adv. Mech. Eng. 2019, 11, 1-11. [CrossRef]

4. Shanghong, H.; Yuwei, X.; Wen, W. Research on Filtering Characteristics of Hydraulic Pulsation Attenuator Based on Bionic Principle of Basilar Membrane of Cochlea. Chin. J. Mech. Eng. 2016, 52, 171-177.

5. Dhakal, R.; Zhou, J.; Palikhe, S.; Bhattarai, K.P. Hydraulic Optimization of Double Chamber Surge Tank Using NSGA-II. Water 2020, 12, 455. [CrossRef]

6. Hwang, H.; Lan, T.; Chen, J. Optimization and Application for Hydraulic Electric Hybrid Vehicle. Energies 2020, 13, 322. [CrossRef]

7. Yang, F.; Deng, B. Filtering performance and optimization of double-chamber compound hydraulic attenuators. Proc. Inst. Mech. Eng. Part C J. Mech. Eng. Sci. 2017, 232, 3250-3262.

8. Abdeljaber, O.; Avci, O.; Kiranyaz, S.; Inman, D.J. Optimization of linear zigzag insert metastructures for low-frequency vibration attenuation using genetic algorithms. Mech. Syst. Signal Process. 2017, 84, $625-641$. [CrossRef]

9. Bravo, T.; Maury, C.; Pinhède, C. Optimisation of micro-perforated cylindrical silencers in linear and nonlinear regimes. J. Sound Vib. 2016, 363, 359-379. [CrossRef]

10. Shang, X.; Zhou, H.; Yang, H. Numerical Study on a Compound Hydraulic Pulsation Attenuator Based on String-Fluid Resonance. J. Mech. Sci. Technol. 2020, 34, 4091-4106. [CrossRef] 
11. Guan, C.; He, S.; Jiao, Z. Development research of reflection-absorption compound type fluid pulsation attenuator. In Proceedings of the IEEE 10th International Conference on Industrial Informatics, Beijing, China, 25-27 July 2012; pp. 606-612.

12. Cai, Y. Fluid Pipeline Dynamics; Zhejiang University Press: Hangzhou, China, 1990.

13. Yang, C.; Lin, M.; Li, D. Improving Steady and Starting Characteristics of Wireless Charging for an AUV Docking System. IEEE J. Ocean. Eng. 2018, 45, 430-441. [CrossRef]

14. Lin, M.; Li, D.; Yang, C. Design of an ICPT system for battery charging applied to underwater docking systems. Ocean Eng. 2017, 145, 373-381. [CrossRef]

15. Zeng, R. Hydraulic Noise Control; Harbin Institute of Technology Press: Harbin, China, 1988.

16. Ichiyanagi, T.; Nishiumi, T. Study on the Insertion Loss Characteristics of Side Branch Resonator in Hydraulic Line. In Proceedings of the JFPS International Symposium on Fluid Power, Toyama, Japan, 15-18 September 2008; pp. 353-358.

17. Shang, X.; Zhou, H.; Xie, A.; Zhu, J. Filtering Characteristics of String Hydraulic Pulsation Attenuator. In Proceedings of the ASME Dynamic Systems and Control Conference, Tysons Corner, VA, USA, 11-13 October 2017; p. 9.

18. Kennedy, J.; Eberhart, R. Particle Swarm Optimization. In Proceedings of the International Conference on Neural Networks, Perth, Australia, 27 November-1 December 1995.

19. Al-Betar, M.A.; Awadallah, M.A.; Faris, H.; Yang, X.S.; Khader, A.T.; Alomari, O.A. Bat-inspired algorithms with natural selection mechanisms for global optimization. Neurocomputing 2018, 273, 448-465. [CrossRef]

20. Lin, M.; Yang, C.; Li, D. Hybrid strategy based model parameter estimation of irregular-shaped underwater vehicles for predicting velocity. Robot. Auton. Syst. 2020, 127, 103480. [CrossRef]

21. Liang, J.J.; Qin, A.K.; Suganthan, P.N.; Baskar, S. Comprehensive learning particle swarm optimizer for global optimization of multimodal functions. IEEE Trans. Evol. Comput. 2006, 10, 281-295. [CrossRef]

22. Du, G.; Zhu, Z.; Gong, X. Fundamentals of Acoustics, 2nd ed.; Nanjing University Press: Nanjing, China, 2001.

Publisher's Note: MDPI stays neutral with regard to jurisdictional claims in published maps and institutional affiliations.

(C) 2020 by the authors. Licensee MDPI, Basel, Switzerland. This article is an open access article distributed under the terms and conditions of the Creative Commons Attribution (CC BY) license (http://creativecommons.org/licenses/by/4.0/). 